\title{
Optimization design and test of rice plug seedling transplanting mechanism of planetary gear train with incomplete eccentric circular gear and non-circular gears
}

\author{
Ye Bingliang ${ }^{1,2^{*}}$, Yi Weiming ${ }^{1,2}$, Yu Gaohong ${ }^{1,2}$, Gao Yang ${ }^{1,2}$, Zhao Xiong ${ }^{1,2}$ \\ (1. Faculty of Mechanical Engineering and Automation, Zhejiang Sci-Tech University, Hangzhou 310018, China; \\ 2. Zhejiang Province Key Laboratory of Transplanting Equipment and Technology, Hangzhou 310018, China)
}

\begin{abstract}
In order to solve the problem of the high-speed mechanized transplanting of rice plug seedlings, the design requirements of transplanting mechanism for rice plug seedlings were analyzed and a kind of rice plug seedling transplanting mechanism of planetary gear train based on the drive with incomplete eccentric circular gear and non-circular gears was designed innovatively. The laboratory kinematics was examined. The working principle of the transplanting mechanism for rice plug seedlings was studied, kinematics analysis of the transplanting mechanism was carried out and its kinematic model was set up. A human-computer interaction optimization method was used to optimize the parameters of the transplanting mechanism. The computer aided analysis and optimization software of the transplanting mechanism based on Visual Basic 6.0 was developed. Through analyzing the influence of design variables on the optimization objectives of the transplanting mechanism, a set of parameters of the transplanting mechanism which meet the requirements of transplanting trajectory and posture for transplanting rice seedlings were obtained by means of human-computer interaction. The structure of the transplanting mechanism was designed according to this set of parameters, and its virtual prototype and physical prototype were set up and manufactured, respectively. The kinematic simulation test and high-speed photography kinematic test of the transplanting mechanism were conducted to obtain its kinematic performances, such as transplanting trajectory and posture. The results of bench test, simulation analysis and theoretical analysis were almost in agreement, which verified the correctness of the theoretical model and design results of the transplanting mechanism, indicating that the optimized transplanting mechanism can satisfy the requirements of transplanting rice seedlings and be applied in the rice plug seedling transplanter.
\end{abstract}

Keywords: plug seedling, transplanting mechanism, incomplete eccentric circular gear, non-circular gear, planetary gear train, optimization design

DOI: $10.25165 /$ j.ijabe.20171006.2712

Citation: Ye B L, Yi W M, Yu G H, Gao Y, Zhao X. Optimization design and test of rice plug seedling transplanting mechanism of planetary gear train with incomplete eccentric circular gear and non-circular gears. Int J Agric \& Biol Eng, 2017; 10(6): 43-55.

\section{Introduction}

In China, rice is the largest planted grain crop and plays an important role in food security. Rice plug

Received date: 2016-07-12 Accepted date: 2017-05-17

Biographies: Yi Weiming, Master student, research interests: agricultural machinery design, Email: 2507244892@qq.com; Yu Gaohong, $\mathrm{PhD}$, Professor, research interests: agricultural machinery design, Email: yugh@zstu.edu.cn; Gao Yang, Master, research interests: agricultural machinery design, Email: 1083097502@qq.com; Zhao Xiong, PhD, Associate Professor, seedling transplanting is currently regarded as the best way to increase yield except the breed improvement. Compared with the mechanized-transplanting for rice blanket seedlings, it has many advantages, such as no

research interests: agricultural machinery design, Email: 59820161@qq.com.

*Corresponding author: Ye Bingliang, $\mathrm{PhD}$, Professor, research interests: agricultural machinery design, Faculty of Mechanical Engineering and Automation, Zhejiang Sci-Tech University, Hangzhou 310018, China. Tel: +86-13336060776, Email: zist_ybl@zstu.edu.cn. 
damage to seedlings, short recovery time, more effective tiller, and high yield. But the research and development work for the rice plug seedling transplanter has progressed very slowly, which has restricted further improvement of the mechanization level of rice cultivation and planting efficiency to a certain extent ${ }^{[1-5]}$. The transplanting mechanism for rice plug seedling possesses complicated kinematics and multiple requirements for work performance, which is the core working part of the rice transplanter that determines the transplanting quality. The automatic rice seedling transplanting machine developed in Japan uses three sets of devices to realize the operations of seedling pick-up, conveying and planting, so the mechanism is of complicate structure and high cost. What's more, the use of semi-rigid plastic plug tray to cultivate seedlings further increases the cost and the requirements of cultivating seedlings. The Japanese machine is not suitable for transplanting rice plug seedlings in China ${ }^{[6-9]}$, so it cannot be applied widely in China. In China, the plug seedling transplanting machine developed by Northeast Agricultural University and Xinhuayu Agricultural Machine Equipment Co., Ltd adopts double-crank mechanism with five bars to complete the above three actions so it only costs $1 / 8$ of the Japanese machine, and the transplanting mechanism is simple and light ${ }^{[10]}$. But the mechanism also has the problems of low efficiency of only 80 plants per minute per row and heavy vibration. It is very difficult to improve transplanting efficiency because of the limitation of the multi-bar mechanism. The seedling pushing angle and angle difference between the seedling pushing angle and seedling pick-up angle are not big enough during the operation of the rice plug seedling transplanting mechanism of the planetary gear train with elliptical gear and incomplete non-circular gear, which was invented by Agricultural Machinery Research Institute of Zhejiang Sci-Tech University in China ${ }^{[11-13]}$. However, the effect of seedling pushing and the upright characteristics of the seedling are not desirable. Therefore, a new type of transplanting mechanism for rice plug seedlings of the planetary gear train with incomplete eccentric circular gear and non-circular gears was proposed, and then the kinematics modeling and analysis, simulation analysis and test of the transplanting mechanism were carried out. This rotary transplanting mechanism with two transplanting arms symmetrically arranged on the gear box can complete the functions of seedling pick-up, conveying and planting, which can increase the motion stability during the working process and transplanting twice in one rotation cycle, and the transplanting efficiency can reach 200 plants per minute per row. Compared with the multi-bar transplanting mechanism in the market, it has the advantages of compact structure, high transplanting efficiency, stable operation and high bearing capacity.

\section{Design requirements and working principle of the transplanting mechanism}

\subsection{Design requirements}

According to the agronomical and technical requirements of transplanting plug seedlings cultivated in plastic plug tray, the transplanting arms of the rice plug seedling transplanter could simulate manual operation to extract the seedlings from the seedling tray and convey them to a certain position, and then push them to the paddy land quickly to finish seedling planting operation. During the operation process, the requirements of not hurting seedlings, high success ratio of seedling pushing and high seedling-standing ratio should be satisfied. In addition, when the transplanting mechanism is running, the transplanting trajectory and posture of the transplanting arms should be good. So the static transplanting trajectory and working process of the seedling transplanting shown in Figure 1 was put forward $^{[11-13]}$. In the transplanting operation, the way of clamping seedling is used to extract seedlings from the tray. That is to say, when the two seedling pick-up claws on the transplanting arm arrive at the surface of the soil bowl, they will clamp the root of the seedling stem to extract the seedling containing soil from the tray at point $C$ and convey it to point $A$ where the two seedling pick-up claws will loosen and the seedling will be pushed and planted in the paddy land under the action of seedling pushing rod. And then the seedling pick-up claws returns through the position $B$ to prepare for the next 
seedling pick-up action. This is a cycle of rice plug seedling transplanting. In addition, in order to avoid the planted seedling is pushed down by the transplanting mechanism when the transplanter is moving forward, the dynamic trajectory of the transplanting mechanism should move upward first and then forward after the seedling pushing.

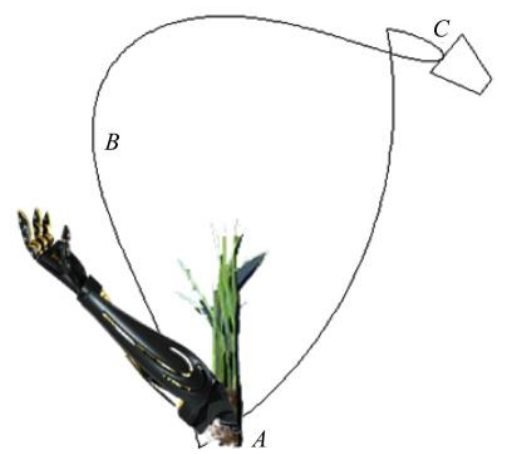

Figure 1 Diagram of the plug seedling transplanting trajectory

\subsection{Composition and work principle}

According to the design requirements of the transplanting mechanism, a new kind of rotary transplanting mechanism for rice plug seedlings called the rice plug seedling transplanting mechanism of the planetary gear train with incomplete eccentric circular gear and non-circular gears shown in Figure 2 was designed. The transplanting mechanism consists of a sun gear of incomplete eccentric circular gear 3 , two middle gears of middle non-circular gear 2 and 4, two planetary gears of planetary non-circular gear1and 5, a convex locking arc 9, two concave locking arcs 7 and 10, a planetary carrier 8 and two transplanting arms 6 and 11 . The rotation center of the sun gear is $O$, the rotation centers of two middle gears are $M_{1}$ and $M_{2}$ and the rotation centers of two planetary gears are $\mathrm{O}_{1}$ and $\mathrm{O}_{2}$, respectively. Point $P$ is the meshing point between the middle gear and sun gear, point $Q$ is the meshing point between the middle gear and planetary gear, and point $G$ is the endpoint of transplanting arm 11. $A B C D E F$ is the transplanting trajectory formed by the endpoint of the transplanting arm.

Because the transplanting mechanism is of the rotational symmetric structure, only one side of the mechanism was considered in the working principle analysis. Sun gear 3 is fixed on the frame, convex locking arc 9 and locking concave arc 10 are fixed on sun gear 3 and middle gear 2, respectively, and transplanting arm 11 is fixed on planetary gear 1 . When the transplanting mechanism is in work, planetary carrier 8 rotates clockwise with a constant speed. If the planetary carrier causes middle gear 2 to mesh with the toothless part of the sun gear, convex locking arc 9 contacts and locks concave arc to avoid the middle gear rotates relative to the planetary carrier while the middle gear may not rotate on its axis, so does the planetary gear. During this process, the trajectory formed by the endpoint of the transplanting arm is $F A B$. If middle gear 2 meshes with the toothed part of the sun gear, the middle gear rotates with a variable speed relative to the planetary carrier, which causes planetary gear 1 to rotates with a variable speed relative to the planetary carrier too. During this process, the trajectory formed by the endpoint of the transplanting arm is $B C D E F$.

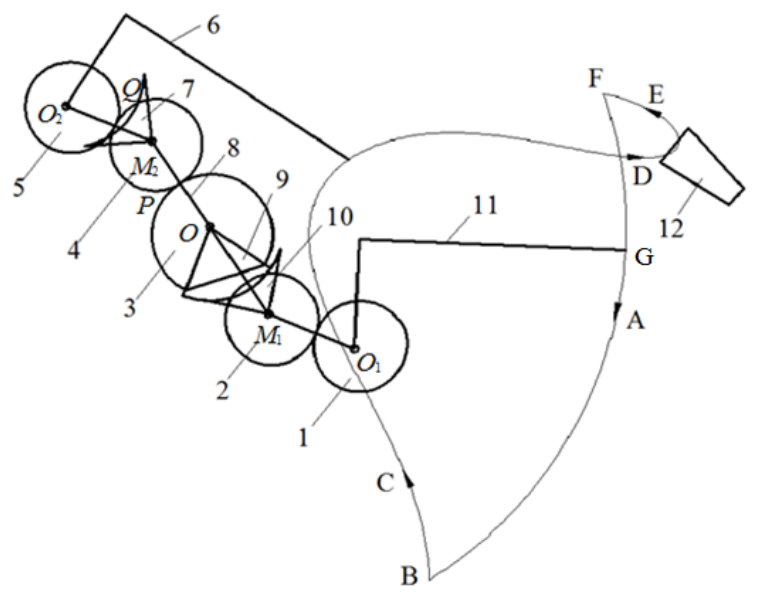

1,5. Planetary non-circular gear 2,4. Middle non-circular gear 3. Incomplete eccentric circular gear 6,11. Transplanting arm 7,10. Concave locking arc 8. Planetary carrier 9. Convex locking arc 12. Plug seedling tray

Figure 2 Transmission diagram of the rice plug seedling transplanting mechanism of the planetary gear train with incomplete eccentric circular gear and non-circular gears

\section{Kinematic model of the transplanting mechanism}

3.1 Kinematic analysis of the transmission with incomplete eccentric circular gear and non-circular gear

As shown in Figure 2, sun gear 3 of the incomplete eccentric circular gear meshes with middle gear 2 of the middle non-circular gear to realize non-uniform intermittent transmission, which is the key for the endpoint of the transplanting arm to form the trajectory 
$A B C D E F$. Figure 3 is the kinematic analysis diagram of the transmission of the incomplete eccentric circular gear and non-circular gear ${ }^{[14-18]}$. As shown in Figure 3, the rectangular coordinate system, $x O y$, was established. For being easy to analyze the motion of this transmission, the middle gear was assumed to rotate counterclockwise. The part of dotted line expresses the initial installation position of the mechanism, and the part of solid line is the position that the planetary carrier rotates by angle $\varphi_{1}$. $O$ is the rotation center of the planet carrier. $O_{1}$ is the geometrical center of the incomplete eccentric circular gear whose pitch curve of toothed part is symmetrical on line $O O_{1} . \quad M_{1}$ is the relative rotation center of the planetary gear. $R$ and $e$ are radius and eccentricity of the incomplete eccentric circular gear, respectively. $R_{1}$ and $\beta$ are radius vector and central angle of the pitch curve of toothed part of the incomplete eccentric circular gear, respectively. $R_{2}$ is radius vector of the pitch curve of the middle gear. $R_{3}$ is radius of the convex locking arc. $\theta$ is the central angle of locking concave arc. $l$ is the center distance between the sun gear and the middle gear. $P$ is the meshing point between the middle gear and the sun gear. When the planetary carrier rotates by angle $\varphi_{1}$, the middle gear rotates by angle $\varphi_{2}$ relative to the planetary carrier.

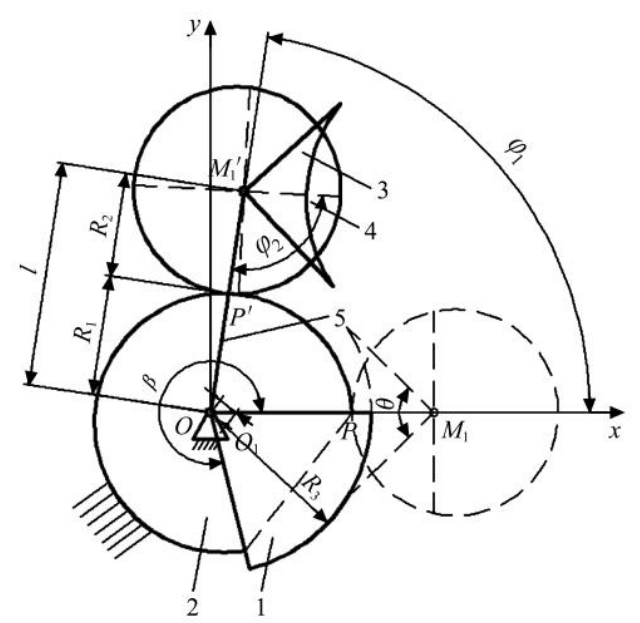

1. Convex locking arc 2. Incomplete eccentric circular gear 3. Concave locking arc 4. Middle non-circular gear 5. Planetary carrier

Figure 3 Kinematic analysis diagram of the transmission with incomplete eccentric circular gear and middle non-circular gear

The distance from meshing point $P$ between the sun gear and middle gear to rotation center $O$ of the planetary carrier is calculated as Equation (1):

$$
\begin{gathered}
R_{1}\left(\varphi_{1}\right)=\sqrt{R^{2}-e^{2} \sin ^{2}\left(\varphi_{1}+\pi-\frac{\beta}{2}\right)}+e \cos \left(\varphi_{1}+\pi-\frac{\beta}{2}\right) \\
0 \leq \varphi_{1} \leq \beta
\end{gathered}
$$

According to the transmission characteristics of the conjugate non-circular gears ${ }^{[19]}$, the perimeter of pitch curve of the middle gear is equal to that of toothed part of the sun gear. When the planetary carrier rotates by angle $\varphi_{1}$ from zero to $\beta$, the middle gear will correspondingly rotate by angle $\varphi_{2}$ from zero to $2 \pi$ relative to the planetary carrier. Therefore, the following equation is obtained.

$$
\int_{0}^{\beta} R_{1}\left(\varphi_{1}\right) \mathrm{d} \varphi_{1}=\int_{0}^{2 \pi} R_{2}\left(\varphi_{2}\right) \mathrm{d} \varphi_{2}
$$

The sum of the pitch curve radius vector of the toothed part of the sun gear and that of middle gear is the center distance of the gear transmission which is calculated as Equation (3):

$$
l=R_{1}\left(\varphi_{1}\right)+R_{2} \varphi_{2}
$$

Therefore, the pitch curve equation of the middle gear is:

$$
R_{2}\left(\varphi_{2}\right)=l-R_{1}\left(\varphi_{1}\right), \quad 0 \leq \varphi_{2} \leq 2 \pi
$$

When the planetary carrier rotates by angle $\mathrm{d} \varphi_{1}$, the middle gear will correspondingly rotate by angle $\mathrm{d} \varphi_{2}$. Therefore, the following equation is obtained.

$$
R_{1}\left(\varphi_{1}\right) \mathrm{d} \varphi_{1}=R_{2}\left(\varphi_{2}\right) \mathrm{d} \varphi_{2}
$$

According to Equations (4) and (5), the angular displacement of the middle gear is calculated as:

$$
\varphi_{2}=\int_{0}^{\varphi_{1}} \frac{R_{1}\left(\varphi_{1}\right)}{l-R_{1}\left(\varphi_{1}\right)} \mathrm{d} \varphi_{1}
$$

To substitute Equation (1) into Equation (4), the following equation is obtained.

$$
\varphi_{2}=\int_{0}^{\varphi_{1}} \frac{\sqrt{R^{2}-e^{2} \sin ^{2} \varphi}+e \cos \varphi}{l-\sqrt{R^{2}-e^{2} \sin ^{2} \varphi}-e \cos \varphi} \mathrm{d} \varphi_{1}
$$

where, $\varphi=\varphi_{1}+\pi-\beta / 2$.

According to Equation (2), when $\varphi_{1}$ is equal to $\beta, \varphi_{2}$ is equal to $2 \pi$. Therefore, the following equation is obtained.

$$
2 \pi=\int_{0}^{\beta} \frac{\sqrt{R^{2}-e^{2} \sin ^{2} \varphi}+e \cos \varphi}{l-\sqrt{R^{2}-e^{2} \sin ^{2} \varphi}-e \cos \varphi} \mathrm{d} \varphi_{1}
$$

Assume $k=e / R$, the following equation is obtained. 


$$
2 \pi=\int_{0}^{\beta} \frac{1-k^{2}}{\frac{l}{R}\left(\sqrt{1-k^{2} \sin ^{2} \varphi}-k \cos \varphi\right)-\left(1-k^{2}\right)} \mathrm{d} \varphi_{1}
$$

The center distance $l$ can be calculated by using Equation (9) and the method of numerical integration.

\subsection{Kinematic analysis of the transmission with} middle non-circular gear and planetary non-circular gear

One side part of the mechanism was considered in the kinematic analysis. The initial installation position of the mechanism is shown in Figure 4a and the mechanism position after the planetary carrier rotated by angle $\varphi_{1}$ is shown in Figure 4b. As shown in Figure 4, Points $O, M_{1}$ and $O_{1}$ are the rotation centers of the sun gear, the middle gear and the planetary gear, respectively. $\delta_{0}$ is the intersection angle between lines $M_{1} O_{1}$ and $M_{1} O$ called the corner of the planetary carrier. When the middle gear and the planetary gear rotate by angle $\varphi_{1}$ along with the planetary carrier, the angular displacement of the middle gear relative to the planetary carrier is $\varphi_{2}$, and that of the planetary gear relative to the planetary carrier is $\varphi_{3}$. Points $P$ and $Q$ are the meshing points between the sun gear and the middle gear, and the middle gear and the planetary gear, respectively. $R_{21}$ is the distance between meshing point $Q$ and rotation center $M_{1}$ of the middle gear, $R_{3}$ is the distance between meshing point $Q$ and rotation center $O_{1}$ of the planetary gear.

The middle gear and the planetary gear are a couple of conjugated non-circular gear. In order to make the transplanting mechanism to be able to make continuous periodic movement, the transmission of the middle gear and the planetary gear should meet the following two conditions. First, the perimeter of the pitch curve of the middle gear is equal to that of the planetary gear; Second, when the angular displacement of the middle gear relative to the planetary carrier $\varphi_{2}$ increases from zero to $2 \pi$, the angular displacement of the planetary gear relative to the planetary carrier $\varphi_{3}$ will increase from zero to $2 \pi$ too. Therefore, the following equations were obtained.

$$
\begin{gathered}
a=R_{21}\left(\varphi_{1}\right)+R_{3}\left(\varphi_{1}\right), \quad 0 \leq \varphi_{1} \leq \beta \\
\varphi_{3}=\int_{0}^{\varphi_{2}} \frac{R_{21}}{R_{3}} \mathrm{~d} \varphi_{2}, \quad 0 \leq \varphi_{2} \leq 2 \pi
\end{gathered}
$$

$$
\varphi_{3}=\int_{0}^{\varphi_{2}} \frac{R_{21}}{a-R_{21}} \mathrm{~d} \varphi_{2}, \quad 0 \leq \varphi_{2} \leq 2 \pi
$$

where, $\alpha$ is the center distance of the transmission of the middle gear and planetary gear.

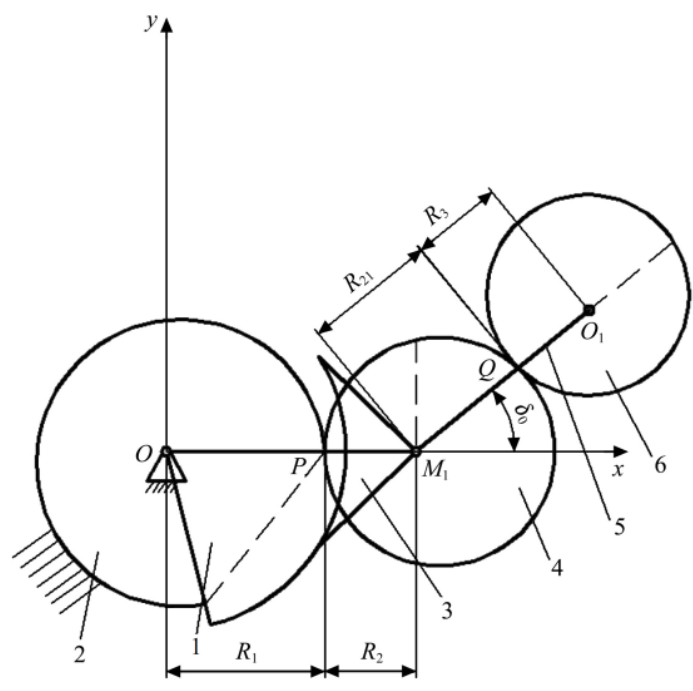

a. Initial installation position of the mechanism

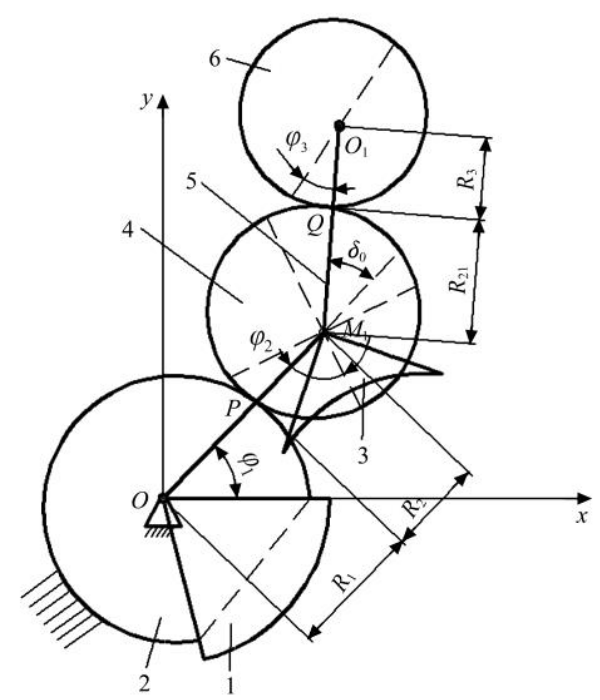

b. Mechanism position after planetary carrier rotated by angle $\varphi_{1}$

1. Convex locking arc 2. Incomplete eccentric circular gear (Sun gear)

3. Concave locking arc 4. Middle non-circular gear (Middle gear) 5. Planetary carrier 6. Planetary non-circular gear (Planetary gear)

Figure 4 Kinematic analysis diagram of the transmission with middle non-circular gear and planetary non-circular gear

Because the middle non-circular gear is symmetrical on the line of $O M_{1}$ in Figure $4 \mathrm{a}$, therefore, $R_{21}\left(\varphi_{1}\right)$ is calculated as Equation (13):

$$
R_{21}\left(\varphi_{1}\right)=R_{2}\left(\varphi_{2}+\pi-\delta_{0}\right)
$$

To determine the value of $R_{21}\left(\varphi_{1}\right)$, the angular displacement of the planetary carrier $\varphi_{1}$ should be obtained firstly when the angular displacement of the middle gear relative to the planetary carrier is equal to $\varphi_{2}+\pi-\delta_{0}$. Because of the difficulty to solve the value 
and the complicate computing, the interpolation method was used to calculate the value $R_{21}\left(\varphi_{1}\right)$ which is a group of discrete values when the angular displacement of the planetary carrier $\varphi_{1}$ increased from zero to $\beta$ with a step of 0.5 .

According to the second condition that the transmission of the middle gear and the planetary gear should meet and Equation (12), the following equation is obtained.

$$
2 \pi=\int_{0}^{2 \pi} \frac{R_{21}}{a-R_{21}} \mathrm{~d} \varphi_{2}
$$

Because $R_{21}\left(\varphi_{1}\right)$ is a group of discrete values, the method of discrete points integration can be used to calculate the center distance $\alpha$. After the values of $R_{21}\left(\varphi_{1}\right)$ and $\alpha$ are obtained, $\varphi_{3}$ can be calculated out according to Equation (12).

The displacement equation of endpoint $G$ of the transplanting arm is:

$$
\left\{\begin{aligned}
X_{G}= & l \cos \left(\varphi_{1}+\varphi_{0}\right)+a \cos \left(\varphi_{1}+\varphi_{0}+\delta_{0}\right)+ \\
& S \cos \left(\alpha_{0}+\varphi_{0}+\delta_{0}+\varphi_{3}+\varphi_{1}\right) \\
Y_{G}= & l \sin \left(\varphi_{1}+\varphi_{0}\right)+a \sin \left(\varphi_{1}+\varphi_{0}+\delta_{0}\right)+ \\
& S \sin \left(\alpha_{0}+\varphi_{0}+\delta_{0}+\varphi_{3}+\varphi_{1}\right)
\end{aligned}\right.
$$

where, $\varphi_{0}$ is the initial installation angle of the planetary carrier; $\alpha_{0}$ is the intersection acute angle between line $O_{1} G$ which is the connection line between the rotation center of the planetary gear and the endpoint of the transplanting arm, and line $M_{1} O_{1}$ of the planetary carrier; $S$ is the distance from rotation center $O_{1}$ of the planetary gear and endpoint $G$ of the transplanting arm as shown in Figure 2.

\subsection{Speed model of the transplanting mechanism}

When the transplanting mechanism for rice plug seedlings is in operation, the planetary carrier rotates clockwise with a constant angular speed $\omega_{1}$. Therefore, the angular speed $\omega_{3}$ of planetary non-circular gear relative to planetary carrier is Equation (16):

$$
\omega_{3}=\left\{\begin{array}{cc}
0 & 0 \leq \varphi_{1}<2 \pi-\beta \\
\frac{R_{1}}{l-R_{1}} \frac{R_{21}}{R_{3}} \omega_{1} & 2 \pi-\beta \leq \varphi_{1}<2 \pi
\end{array}\right.
$$

According to the derivation of Equation (15), the velocity equation of endpoint $G$ of the transplanting arm is:

$$
\left\{\begin{aligned}
\dot{X}_{G}= & -\omega_{1} l \sin \left(\varphi_{1}+\varphi_{0}\right)-a \omega_{1} \cos \left(\varphi_{1}+\varphi_{0}+\delta_{0}\right)- \\
& S\left(\omega_{1}+\omega_{3}\right) \sin \left(\alpha_{0}+\varphi_{0}+\delta_{0}+\varphi_{3}+\varphi_{1}\right) \\
\dot{Y}_{G}= & \omega_{1} l \cos \left(\varphi_{1}+\varphi_{0}\right)+a \omega_{1} \cos \left(\varphi_{1}+\varphi_{0}+\delta_{0}\right)+ \\
& S\left(\omega_{1}+\omega_{3}\right) \cos \left(\alpha_{0}+\varphi_{0}+\delta_{0}+\varphi_{3}+\varphi_{1}\right)
\end{aligned}\right.
$$

Therefore, the resultant velocity of endpoint $G$ of the transplanting arm is Equation (18):

$$
v_{G}=\sqrt{\dot{X}_{G}^{2}+\dot{Y}_{G}^{2}}
$$

\section{Parameter optimization of the transplanting mechanism}

Parameter optimization of the transplanting mechanism for rice plug seedlings is a complex fuzzy problem with multiple objectives, strong coupling and nonlinear constraint, therefore, a human-computer interaction optimization method was applied to optimize the kinematic parameters of the transplanting mechanism. The optimization general idea is to establish the optimization design mathematical model including the above-mentioned kinematic model of the transplanting mechanism, and develop the computer aided analysis and optimization software for the parameter optimization of the mechanism. Then the software is applied to analyze the influences of the design variable on the optimization objectives. Finally, the human-computer interaction mode is adopted to realize parameter optimization of the mechanism by means of the experts' experience and analyze the optimization results ${ }^{[11,20-23]}$.

\subsection{Optimization objectives and design variables}

Endpoint $G$ of the transplanting arm of the rice plug seedling transplanting mechanism of the planetary gear train with incomplete eccentric circular gear and non-circular gears realizes the transplanting trajectory as shown in Figure 2 to meet the requirements of transplanting rice plug seedlings, the motion interference, static and dynamic kinematic trajectory, and the posture of the transplanting arm of the transplanting mechanism should be taken into comprehensive consideration during the optimization process. The optimization objectives of the mechanism are the followings.

1) There is no interference between the transplanting arm and the plug seedling tray, and between the two arms.

2) The trajectory of seedling pick-up for the seedling 
claw to approach the plug seedling tray should be below that for the seedling claw to leave the tray with the seedling, which means that the trajectory of seedling pick-up should form a ring.

3) The width and height of the ring should be not less than $28 \mathrm{~mm}$ and $25 \mathrm{~mm}$, respectively.

4) At the end of seedling pick-up while the seedling pick-up claw clamps the seedling, the value of the seedling pick-up angle which is the intersection acute angle between the transplanting arm and the horizontal line should be from $5^{\circ}$ to $15^{\circ}$. At the start of seedling pushing, the value of the seedling pushing angle which is the intersection acute angle between the transplanting arm and the horizontal line should be from $45^{\circ}$ to $65^{\circ}$. The angle difference between the seedling pick-up angle and seedling pushing angle should be about $50^{\circ}$.

5) The height of the transplanting static trajectory should be greater than $260 \mathrm{~mm}$.

6) During the process of seedling pushing, if the width of the opening part between the dynamic trajectory and the ground is too wide, the planted seedling will be easy to get flattened or be floating, therefore, the width of the opening part should be less than $30 \mathrm{~mm}$.

7) The operation of the seedling pushing should be completed before the mechanism returns, and the transplanting arm should move upward after seedlings pushing to avoid the planted seedlings are pushed down.

According to the kinematics analysis and the optimization objectives, the optimization design variables were decided as: the radius and eccentric distance of the incomplete eccentric circular gear $R$ and $e$, the central angle of the pitch curve of toothed part of the incomplete eccentric circular gear $\beta$, the intersection acute angle between line $O_{1} G$ and $M_{1} O_{1}$ part of the planetary carrier $\alpha_{0}$, the corner of the planetary carrier $\delta_{0}$, the initial installation angle of the planetary carrier $\varphi_{0}$, the distance $S$ between rotation center $O_{1}$ of the planetary gear and endpoint $G$ of the transplanting arm.

\subsection{Computer aided analysis and optimization software of the transplanting mechanism}

Base on the visualized platform of Visual Basic 6.0, a computer aided analysis and optimization software of the transplanting mechanism was developed. Figure 5 is the interface of the transplanting trajectory analysis and optimization. By using the software, the followings can be realized, such as the influence analysis of the design variables on the transplanting trajectory, the motion interference checking of the mechanism, parameters confirmation of the incomplete eccentric circular gear and non-circular gear, values output of the seedling pick-up angle, the seedling pushing angle, the angle difference, the trajectory height, the rotated angle of the planetary carrier, and the angular displacement of the four non-circular gears relative to the planetary carrier, etc.

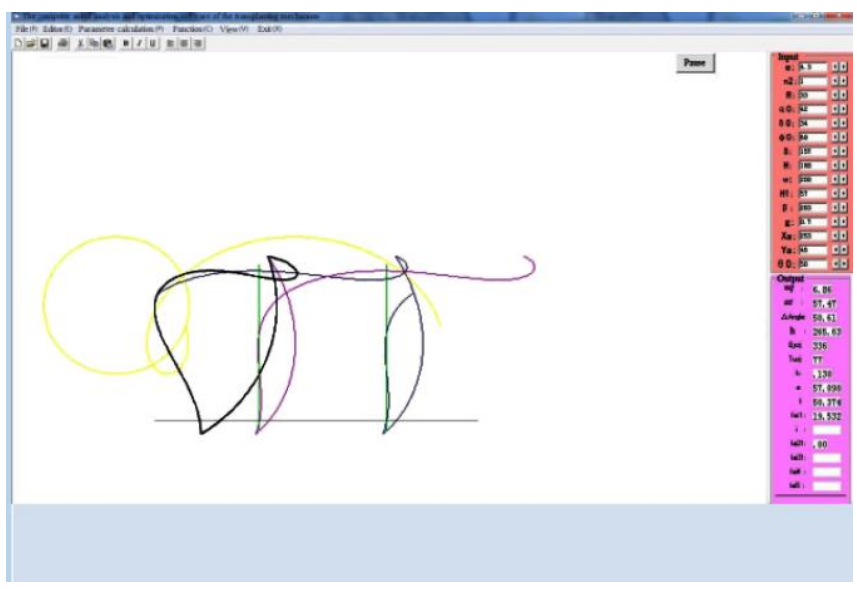

Figure 5 Interface of the transplanting trajectory analysis and optimization

\subsection{Influences analysis of design variables on optimization objectives}

The influences of the design variables on the transplanting static trajectory are shown in Figure 6. The rectangular coordinate system was set up taking the rotation center of the sun gear as the coordinate origin, $x$ and $y$ axes represent the coordinates of the $x$ and $y$ directions of the trajectory, respectively (Unit: $\mathrm{mm}$ ).

As shown in Figure 6a, the radius of the incomplete eccentric circular gear $R$ only affects the shape of the transplanting trajectory and has no influence on its upright characteristics. If the value of $R$ increases, the trajectory will become fat and high, and the width of the opening part becomes bigger. When the radius increases to a certain value, the ring in the upper right part of the trajectory disappears.

As shown in Figure 6b, the eccentric distance of the incomplete eccentric circular gear $e$ affects the shape of the transplanting trajectory. If the value of $e$ increases, 
the trajectory becomes thinner and a little shorter, the width of the opening part becomes narrower, and the ring shape appears gradually.

As shown in Figure 6c, the central angle of the pitch curve of toothed part of the incomplete eccentric circular gear $\beta$ affects the size of the middle gear and the bending degree of its pitch curve, the shape and the upright characteristics of the trajectory, and determines the

stopping time of the planetary gear relative to the planetary carrier of the transplanting mechanism. If the value of $\beta$ increases, the upper half section of the trajectory rotates clockwise and its height becomes lower, the working trajectory section while the convex locking arc is in use becomes shorter, the ring becomes thinner and narrower, and the width of the opening part becomes a little bigger.

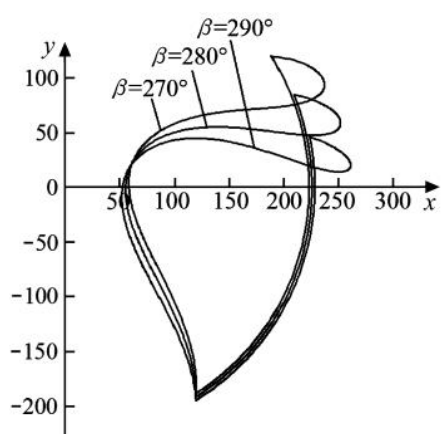

c. Different values of $\beta$

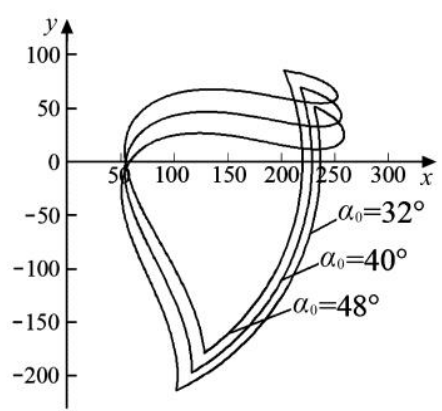

d. Different values of $\alpha_{0}$

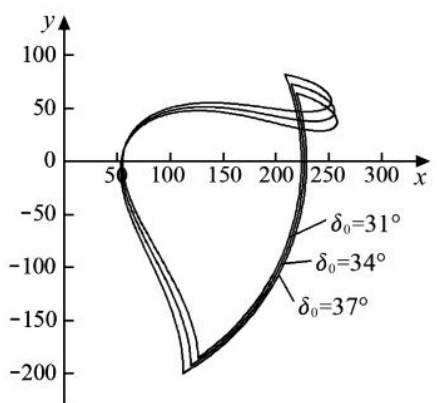

e. Different values of $\delta_{0}$

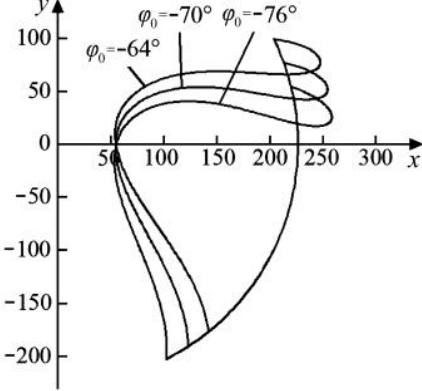

f. Different values of $\varphi_{0}$

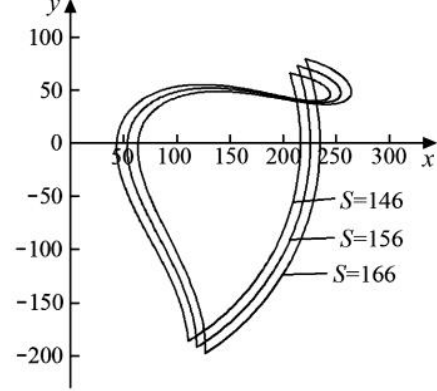

g. Different values of $S$

Figure 6 Influence of design variables on the transplanting trajectory

As shown in Figure 6d, the intersection acute angle between line $O_{1} G$ and $M_{1} O_{1}$ part of the planetary carrier $\alpha_{0}$ affects not only the trajectory shape but also its upright characteristics, the seedling pick-up angle and seedling pushing angle. If the value of $\alpha_{0}$ decreases, the position of the trajectory becomes lower, the width of the ring becomes smaller, the height of the ring becomes bigger, and the whole trajectory will incline slightly. If the value of $\alpha_{0}$ increases, the influence of design variable $\alpha_{0}$ on the trajectory is just opposite to the above.

As shown in Figure 6e, the corner of the planetary carrier $\delta_{0}$ affects the upright characteristics and shape of the trajectory and further causes the change of the seedling pick-up angle and seedling pushing angle. If the value of $\delta_{0}$ increases, the whole trajectory rotates counterclockwise, its position has the trend of moving upward and the ring of the trajectory doesn't change obviously.

As shown in Figure 6f, the initial installation angle of the planetary carrier $\varphi_{0}$ only affects the upright characteristics of the trajectory and further causes the change of the seedling pick-up angle and seedling pushing angle, not the trajectory shape. If the absolute value of $\varphi_{0}$ increases, the whole trajectory rotates clockwise and moves downward. The position of the trajectory moves from the condition of upright to tilting, and the width of the opening part doesn't change nearly.

As shown in Figure $6 \mathrm{~g}$, if the distance $S$ between rotation center $O_{1}$ of the planetary gear and endpoint $G$ of the transplanting arm increases, the whole trajectory moves right, the working trajectory section while the convex locking arc is in use becomes longer, the height of the ring becomes bigger and its width becomes a little bigger too, while the trajectory becomes higher and the 
width of opening part becomes narrower.

Beside the seven parameters above mentioned, the value of the plant spacing $H$ has great influence on the width of the opening part. If the plant spacing is bigger, the width of opening part becomes narrower.

\subsection{Optimization results}

Base on the influence analysis of design variables on the optimization objectives and the comprehensive consideration of the influences of parameters, the developed software was used to obtain a set of parameters: $R=33 \mathrm{~mm}, e=4.3 \mathrm{~mm}, \beta=281^{\circ}, \alpha_{0}=42^{\circ}, \delta_{0}=35^{\circ}, \varphi_{0}=69^{\circ}$, and $S=155 \mathrm{~mm}$. After optimization, the height of the transplanting trajectory is equal to $278.39 \mathrm{~mm}$, the width and height of the ring are $34 \mathrm{~mm}$ and $34.56 \mathrm{~mm}$, respectively, the seedling pick-up angle $\alpha_{q}$ is $4.37^{\circ}$, the seedling pushing angle $\alpha_{t}$ is $56.77^{\circ}$, the angle difference $\Delta \alpha$ is $52.4^{\circ}$, the plant spacing $H$ is $180 \mathrm{~mm}$, and the width of the opening part $L$ is $15.63 \mathrm{~mm}$. The trajectory of the endpoint of the transplanting arm is shown in Figure 7.

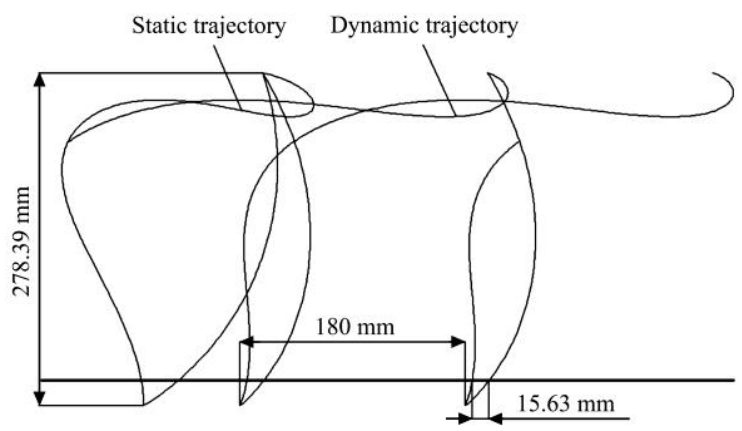

Figure 7 Transplanting trajectory after optimization

The resultant velocity curve as shown in Figure 8 was obtained through the theoretical analysis while the rotation speed of the transplanting mechanism was $100 \mathrm{r} / \mathrm{min}$. As shown in the Figure, the position (1) is the position of seedling pushing, the position (2) is the position of seedling pick-up. The absolute value of the velocity of the transplanting arm endpoint is very small when the mechanism is at the time of seedling pick-up,

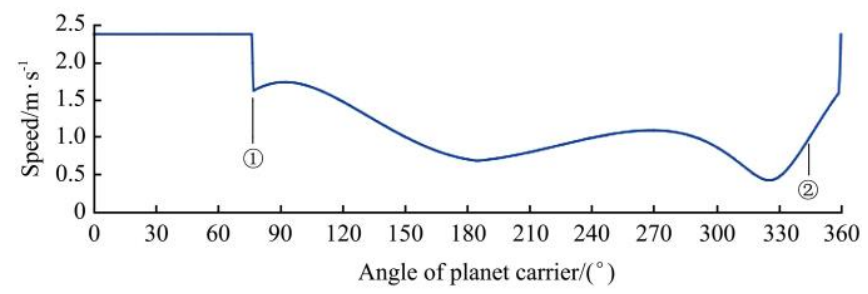

Figure 8 Resultant velocity curve of the endpoint of the transplanting arm but it is greater when the mechanism is at the time of seedling pushing, which can ensure high accuracy and success ratio of seedling pick-up, and to push out seedlings smoothly.

\section{Motion simulation of the transplanting mechanism}

The optimized parameters was used to complete the structure design of the transplanting mechanism, and the three-dimensional modeling software UG8.0 to create the three-dimensional solid model of the mechanism to conduct virtual assembly, then the assembly shown in Figure 9 was imported into ADAMS2010 to conduct the motion simulation analysis. The kinematic trajectory and velocity curves of the transplanting arm endpoint were shown in Figures 10b, 12c and 12d respectively.

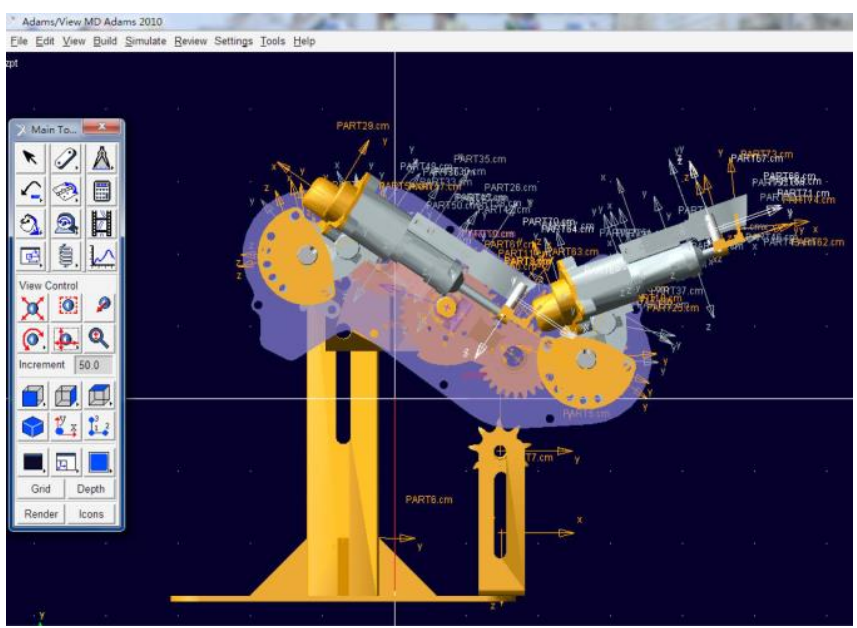

Figure 9 Virtual prototype of the transplanting

\section{Prototype test of the transplanting mechanism}

The prototype was manufactured and installed on the test bench for carrying out kinematic test. The kinematic trajectory and velocity curves of the transplanting arm endpoint, and the posture of the transplanting arm were obtained and compared with the results of the theoretical analysis and simulation analysis, through which the correctness of the theoretical model and design of the mechanism can be verified, and whether the designed mechanism can satisfy the transplanting requirements can be checked.

\subsection{Test procedures}

According to the following procedures, the high speed camera (type VW-6000/5000), picture controller, 
Blaster's MAS image analysis software, and test bench were used to conduct kinematic tests of the mechanism.

1) Install the mechanism on the test bench and adjust it to the suitable position.

2) Mark a white point on the endpoint of the transplanting arm for catching the kinematic trajectory of the endpoint.

3) Install the high speed camera and ensure its lens right face the side of the transplanting mechanism, then open the camera and set the shooting frame rate is 370 frames per second.

4) Adjust the light intensity to ensure the mechanism and the white marked point can be displayed cleanly in the screen.

5) Start the bench and adjust the rotation speed of the AC servo motor to $100 \mathrm{r} / \mathrm{min}$. After the mechanism runs steadily, collect the images.

6) Use the Blaster's MAS image analysis software to deal with and analyze the data.

\subsection{Analysis of test results}

The kinematic trajectory of the transplanting arm endpoint (Figure 10a), the angular displacement of the transplanting arm (Figure 11a) and the velocity curves of $x$ and $y$ directions of the transplanting arm endpoint (respectively shown in Figures $12 \mathrm{a}$ and 12b) were obtained through the bench test. The test results and theoretical results for the seedling pushing angle and seedling pick-up angle, and the angle difference and the relative theoretical results of the other transplanting mechanism called the rice plug seedling transplanting mechanism of the planetary gear train with elliptical gears and incomplete non-circular gear are shown in Table 1. The following conclusions can be drawn through comparisons of the above results shown in Figures 10-12 and Table 1.

1) The kinematic trajectory and velocity curves obtained in the bench test, theoretical analysis and simulation analysis are almost identical. But in order to reduce the impact on the mechanism when the locking arcs disengage from each other, a set of buffer device was added in the virtual prototype and physical prototype, which causes the difference on the kinematic trajectory and velocity curves between the theoretical analysis, and the bench test and simulation analysis when the buffer device is in use. For example, there exists a sharp point on the theoretical trajectory, whereas an arc on the trajectory of the simulation analysis and bench test. What's more, comparing with the theoretical velocity curve, there exist some fluctuations on the simulation velocity curve. This difference mainly results from that most inner components of the transplanting arms and the meshing movement of the gears in the mechanism adopt collision constraint, and the motions from the locking arcs engagement to the buffer device engagement, and then to gear meshing are not very stable. The main reasons for the fluctuations of the bench test curve are the follows: a) There exist errors in the components machining and mechanism assembly. b) The chain transmission in test causes vibration. c) The rotation speed of the electric motor is not constant, fluctuating from $95 \mathrm{r} / \mathrm{min}$ to $105 \mathrm{r} / \mathrm{min}$. d) That some marked points are not clear causes the error of picking the points.

2) The angular displacement curves of the transplanting arm of the bench test and theoretical analysis are basically the same. The reason for the difference is as same as the above. Compare with the relative theoretical results of another transplanting mechanism, the seedling pushing angle and angle difference of the transplanting mechanism designed in this paper are both greater, which is benefit to increase success ratio of the seedling pushing and upright seedling ratio.

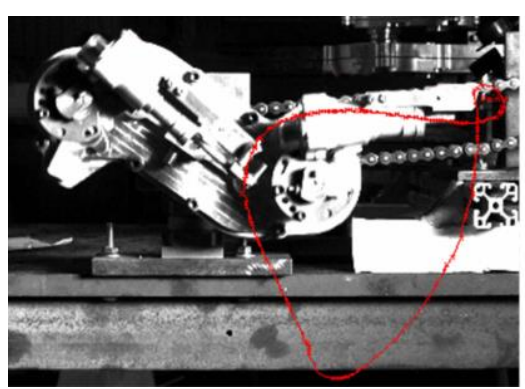

a. Bench test trajectory

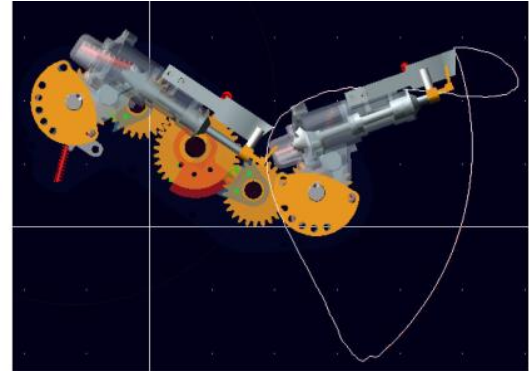

b. Simulation analysis trajectory

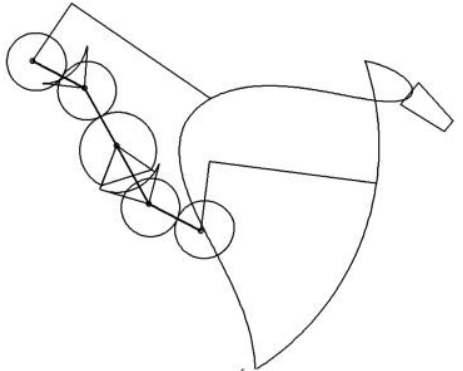

c. Theoretical analysis trajectory

Figure 10 Comparison of the kinematic trajectory of the transplanting arm endpoint 


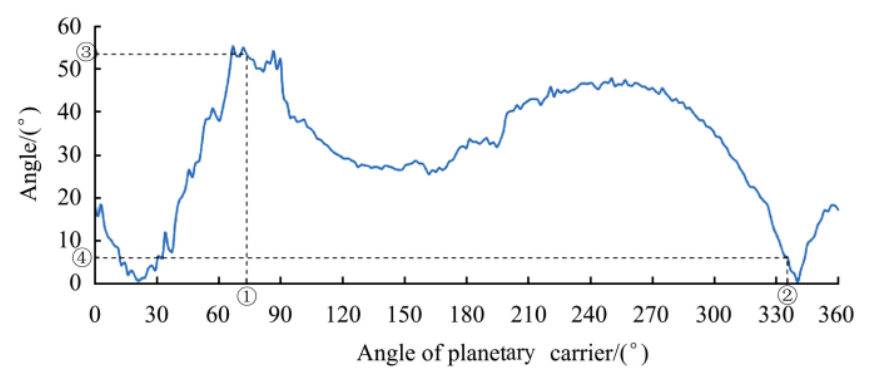

a. Bench test curve

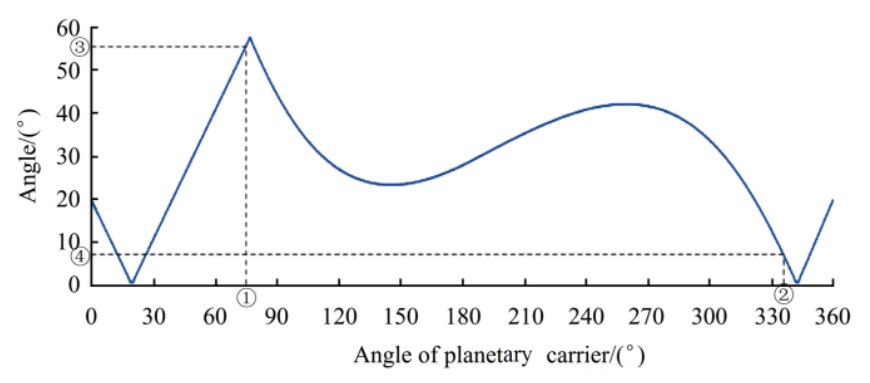

b. Theoretical analysis curve

Notee: (1) Seedling pushing position; (2) Seedling pick-up position; (3) Seedling pushing angle; (4) Seedling pick-up angle.

Figure 11 Comparison of the angular displacement curve of the transplanting arm
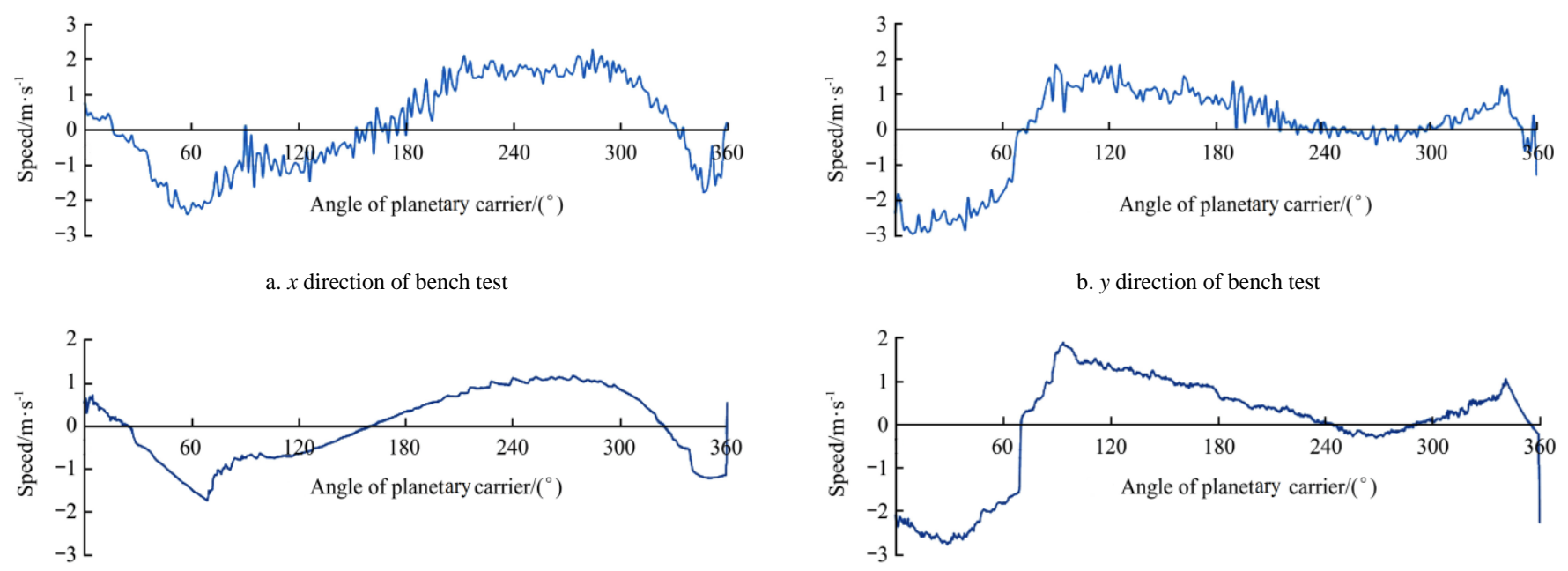

c. $x$ direction of simulation analysis

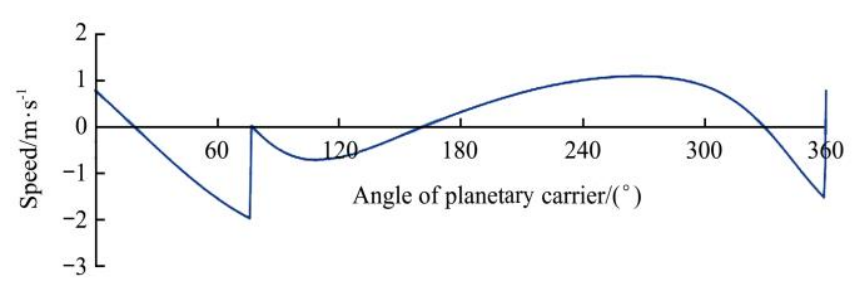

e. $x$ direction of theoretical analysis

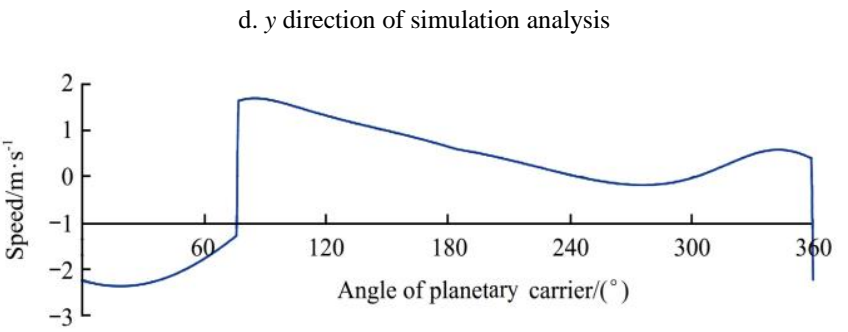

f. $y$ direction of theoretical analysis

Figure 12 Comparison of the $x$ and $y$ direction velocity of the transplanting arm endpoint

Table 1 Comparison of seedling pick-up angle, seedling pushing angle and the angle difference

\begin{tabular}{ccccc}
\hline Parameters & Test results & Theoretical results & Difference & Theoretical results of another transplanting mechanism \\
\hline Seedling pushing angle $/\left(^{\circ}\right)$ & 55.23 & 56.77 & -1.54 & 56.27 \\
Seedling pick-up angle $/\left(^{\circ}\right)$ & 4.62 & 4.37 & 0.25 & 8.83 \\
Angle difference $/\left(^{\circ}\right)$ & 50.61 & 52.40 & 1.79 & 47.44 \\
\hline
\end{tabular}

3) The prototype bench test can prove the theoretical model and design of the transplanting mechanism is correct, the kinematic trajectory and transplanting arm posture of the mechanism can meet the requirements of rice plug seedling transplanting.

\section{Conclusions}

1) Based on the design requirements analysis of the transplanting mechanism for rice plug seedlings, a new kind of rice plug seedling transplanting mechanism of the planetary gear train with incomplete eccentric circular gear and non-circular gears was put forward in this study. The working principle of the transplanting mechanism was studied, the kinematics analysis of the transplanting mechanism was carried out and the kinematics model of the transplanting mechanism was set up. 
2) The human-computer interaction optimization method was used to optimize the parameters of the transplanting mechanism. The computer aided analysis and optimization software of the transplanting mechanism was developed, the influence of design parameters on the optimization objectives was analyzed, and then a group of better parameters satisfying the working requirements of the rice seedling transplanting were obtained: the radius of the incomplete eccentric circular gear $R=33 \mathrm{~mm}$, the eccentric distance of the incomplete eccentric circular gear $e=4.3 \mathrm{~mm}$, the central angle of the pitch curve of toothed part of the incomplete eccentric circular gear $\beta=281^{\circ}$, the intersection acute angle between line $O_{1} G$ and $M_{1} O_{1}$ part of the planetary carrier $\alpha_{0}=42^{\circ}$, the corner of the planetary carrier $\delta_{0}=35^{\circ}$, the initial installation angle of the planetary carrier $\varphi_{0}=69^{\circ}$, the distance between rotational center $O_{1}$ of the planetary gear and endpoint $G$ of the transplanting arm $S=155 \mathrm{~mm}$.

3) The comparison of results among the theoretical analysis, the virtual simulation and the high speed photography kinematic test shows that the theoretical model and design of the transplanting mechanism are correct, and the optimized transplanting mechanism can be applied in a transplanter for rice plug seedlings.

\section{Acknowledgments}

The authors thank the editors and the anonymous reviewers whose constructive comments have notably contributed to the improved quality and clarity of this paper. This research was supported in part by the Natural Science Foundation of China (Grant No. 51275478 and 51575495), the Zhejiang Provincial Natural Science Foundation of China (Grant No. LZ16E050003 and LY15E050025), the 521 Talent Cultivation Plan, the Innovative Research Team Funded Projects and the Science Foundation of Zhejiang Sci-Tech University (Grant No. 15022011-Y).

\section{[References]}

[1] Li J Q, Zhang G F, Chen J N, Zhao Y. Developments and prospects of application of the ordered throwing mechanism for plotted rice-seedling. Agricultural Mechanization Research, 2008; 2: 1-5. ( in Chinese)
[2] Song J N, Zhuang N S, Wang L C, Liu X W, Wei W J. The rice planting mechanization development direction in China in the 21st century. Journal of China Agricultural University, 2000; 5(2): 30-33. ( in Chinese)

[3] Edathiparambil V T. Development of a mechanism for transplanting rice seedlings. Mechanism and Machine Theory, 2002; 37: 395-410.

[4] Guo L S, Zhang W J. Kinematic analysis of a rice transplanting mechanism with eccentric planetary gear train. Mechanism Machine Theory, 2001; 36(11): 1175-1188.

[5] Yuan X B, Zhang G F, Chen J N, Zhao Y. Development on rice plotted-seedling sequential transplanter of ejection type. Journal of Zhejiang Sci-Tech University, 2011; 28(5): 749-753. (in Chinese)

[6] Naokatsu I, Shuichi K, Shunro N. Transplanting machine. Chinese Patent, ZL99118740.7, 2000-05-03. (in Chinese)

[7] Bao B Q, An L Z, Hu W Y. The paddy field mechanization state in Japan and China. Agricultural Mechanization Research, 2002; 3: 23-25. (in Chinese)

[8] Li Y M, Xu L Z, Xiang Z G, Deng L L. Research advances of rice planting mechanization in Japan. Transactions of the CSAE, 2005; 21(11): 182-185. (in Chinese)

[9] Bao C J, Li B. The research progress of rice transplanter in Japan. Transactions of the CSAM, 2004; 35(1): 162-166. (in Chinese)

[10] Yu X X, Zhao Y, Chen B C, Zhou M L, Zhang H, Zhang Z C. Current situation and prospect of transplanter. Transactions of the CSAM, 2014; 45(8): 44-53. ( in Chinese)

[11] Yu G H, Huang X Y, Ye B L, Hu H J, Yu T F. Principle analysis and parameters optimization of rotary rice pot seedling transplanting mechanism. Transactions of the CSAE, 2013; 29(3): 16-22. (in Chinese)

[12] Ye B L, Jin X J, Yu G H, Li L, Gao Y, Zhu H. Parameter modification guiding optimization design and tests of a rotary transplanting mechanism for rice plug seedlings. Applied Engineering in Agriculture, 2015; 31(6): 863-873.

[13] Ye B L, Wu G H, Yu G H, Li L. Design and Test on Transplanting Arm of Rotary Transplanting Mechanism for Rice Pot-seedling. Transactions of the CSAM, 2015; 45(7): 45-52. (in Chinese)

[14] Yu G H, Qian M B, Zhao Y, Wu C Y. Analysis of kinematic principle of transplanting mechanism with eccentric gears and non-circular gears. Transactions of the CSAM, 2009; 40(3): 81-84. (in Chinese)

[15] Zhao Y. Analysis and Synthesis of Agricultural Machinery. Beijing, China: China Machinery Press, 2009. (in Chinese)

[16] Yu G H, Yu T F, Ye B L, Jia D B, Wang L W, Hu H J. Research on a new planetary gear train mechanism. Chinese Journal of Mechanical Engineering, 2013; 49(15): 55-61. (in 
Chinese)

[17] Zhao Y, Zhao X, Zhang W W, Dai L. Modern design theory and method of rice transplanter. Transactions of the CSAM, 2011; 42(3): 65-68, 43. (in Chinese)

[18] Ye B L, Yu G H, Chen Z W, Zhao Y. Kinematics modeling and parameters optimization of seedling pick-up mechanism of planetary gear train and non-circular gear. Transactions of the CSAE, 2011; 27(12): 7-12. (in Chinese)

[19] Yao W X. Noncircular Gear Design. Beijing, China: China Machinery Press, 2013. (in Chinese)

[20] Teng H F, Wang Y H, Shi Y J. Key supporting techniques of human-computer cooperation. Chinese Journal of Mechanical Engineering, 2006; 42(11): 1-9. (in Chinese)
[21] Wu C Y, Zhao Y, Chen J N. Optimization design of rice transplanter separating-planting mechanism with visualization human-computer interaction method. Transactions of the CSAM, 2008; 39(1): 46-49. (in Chinese)

[22] Ye B L, Liu A, Yu G H, Luo C X. Parameters optimization with human-computer interaction method and experiment of vegetable seedling pick-up mechanism. Transactions of the CSAM, 2013; 44(2): 57-62. (in Chinese)

[23] Yu G H, Chen Z W, Zhao Y, Sun L, Ye B L. Study on vegetable plug seedling pick-up mechanism of planetary gear train with ellipse gears and incomplete non-circular gear. Chinese Journal of Mechanical Engineering, 2012; 48(13): 32-39. (in Chinese) 